\title{
RANK-ORDERING STATISTICS OF EXTREME EVENTS: APPLICATION TO THE DISTRIBUTION OF LARGE EARTHQUAKES
}

\author{
Didier Sornette $^{1}$, Leon Knopoff ${ }^{2,3}$, Yan Kagan ${ }^{3}$ and Christian Vanneste ${ }^{1}$ \\ ${ }^{1}$ Laboratoire de Physique de la Matière Condensée, CNRS URA 190 \\ Université de Nice-Sophia Antipolis, B.P. 71 \\ Parc Valrose, 06108 Nice Cedex 2, France \\ ${ }^{2}$ Department of Physics, University of California Los Angeles, California 90095 and \\ ${ }^{3}$ Institute of Geophysics and Planetary Physics \\ University of California, Los Angeles, California 90095-1567
}

\begin{abstract}
Rank-ordering statistics provides a perspective on the rare, largest elements of a population, whereas the statistics of cumulative distributions are dominated by the more numerous small events. The exponent of a power law distribution can be determined with good accuracy by rank-ordering statistics from the observation of only a few tens of the largest events. Using analytical results and synthetic tests, we quantify the systematic and the random errors.

We also study the case of a distribution defined by two branches, each having a power law distribution, one defined for the largest events and the other for smaller events, with application to the World-Wide (Harvard) and Southern California earthquake catalogs. In the case of the Harvard moment catalog, we make more precise earlier claims of the existence of a transition of the earthquake magnitude distribution between small and large earthquakes; the $b$-values are $b_{2}=2.3 \pm 0.3$ for large shallow earthquakes and $b_{1}=1.00 \pm 0.02$ for smaller shallow earthquakes. However, the cross-over magnitude between the two distributions is ill-defined. The data available at present do not provide a strong constraint on the cross-over which has a $50 \%$ probability of being between magnitudes 7.1 and 7.6 for shallow earthquakes; this interval may be too conservatively estimated. Thus, any influence of a universal geometry of rupture on the distribution of earthquakes world-wide is ill-defined at best. We caution that there is no direct evidence to confirm the hypothesis that the
\end{abstract}


large-moment branch is indeed a power law. In fact, a gamma distribution fits the entire suite of earthquake moments from the smallest to the largest satisfactorily. There is no evidence that the earthquakes of the Southern California catalog have a distribution with two branches, or that a rolloff in the distribution is needed; for this catalog $b=1.00 \pm 0.02$ up to the largest magnitude observed, $M_{W} \simeq 7.5$; hence we conclude that the thickness of the seismogenic layer has no observable influence whatsoever on the frequency distribution in this region. 


\section{Introduction}

The observation that many natural phenomena have size distributions that are power laws, has been taken as a fundamental indication of an underlying self-similarity [Mandelbrot, 1983; Aharony and Feder, 1989; Riste and Sherrington, 1991]. A power law distribution indicates the absence of a characteristic size and as a consequence that there is no upper limit on the size of events. The largest events of a power law distribution completely dominate the underlying physical process; for instance, fluid-driven erosion is dominated by the largest floods and most deformation at plate boundaries takes place through the agency of the largest earthquakes. It is a matter of debate whether power law distributions, which are valid descriptions of the numerous small and intermediate events, can be extrapolated to large events; the largest events are, almost by definition, undersampled. Based on analytical calcu-

lations and synthetic tests, we demonstrate that the observation of a few tens of the largest events is sufficient to qualify their distribution with good precision, using the rank-ordering technique, initially introduced in linguistics [Zipf, 1949] and later largely used in statistics [Epstein and Sobel, 1953; Gumbel, 1960; Deemer and Votaw, $1955]$.

In the case of earthquakes, a number of authors have proposed a geometrical argument that the distribution for large earthquakes should be substantially different from that for small ones because of the one-dimensional character of the fracture surface of large earthquakes and its two-dimensional character for small earthquakes [Kanamori and Anderson, 1975; Geller, 1976; Shimazaki, 1986; Rundle, 1989; Scholz, 1990; Romanowicz, 1992; Pacheco et al., 1992; Romanowicz and Rundle, 1993; Okal and Romanowicz, 1994]. More specifically they propose that the large-magnitude branch of the distribution is also a power law and that the cross-over moment or magnitude between these two distributions is a measure of the thickness of the seismogenic zone in the case of strike-slip earthquakes and of the downdip dimension of rupture in the case of earthquakes in subduction zones. Pacheco et al. [1992] claim to have identified a kink in the distribution of shallow transform fault earthquakes around magnitude 5.9 to 6.0 , which corresponds to a characteristic dimension of about $10 \mathrm{~km}$; a kink for subduction zones is presumed to occur at a moment magnitude near 7.5, which corresponds to a down-dip dimension of the order of $60 \mathrm{~km}$. 
It is possible to show that the power-law distribution that holds for small earthquakes cannot be extended to infinity because it would imply that an infinite amount of energy be released from the earth's interior [Knopoff and Kagan, 1977; Kagan and Knopoff, 1984; Kagan, 1994]. According to either the energy or the geometrical arguments, there must be a cross-over or a rollover to a second branch of the distribution. According to the energy argument, a truncation of the distribution for small earthquakes is not forbidden; there is however the following intuitive reason why a cutoff should not be abrupt: we see no reason why the distribution should be finite for a magnitude of let us say 7.99, and zero for magnitude 8.00 [Kagan and Knopoff, 1984]. Although there is no compelling argument that the branch of the distribution for earthquakes with large energies must be a power law, it is clear that if this distribution is indeed a power law, then it must have an exponent that is larger than that for smaller earthquakes; specifically, the energy in the power law cumulative distribution of large earthquakes should fall off as $E^{-b / \beta}$ with $b / \beta>1$ [Knopoff and Kagan, 1977], where $b$ is the usual exponent in the magnitude-frequency law and $\beta$ is generally taken to be 1.5 .

We analyze the problem of the statistics of power law distributions in detail. We pay special attention to the case of a distribution composed of two power laws, one for the extreme events and the other for intermediate and small events. We find that a modest amount of data for the largest events is sufficient to determine the exponent for this branch, whereas the value of the cross-over is ill-defined, unless one has data for a much larger number of large magnitude events. We apply this technique to the analysis of the Harvard catalog [Dziewonski et al., 1993] of worldwide earthquake moments and to the Southern California earthquake catalog [Hutton and Jones, 1993].

Consider a stochastic process in which the outcome, which we call the energy $E$ generically, has the distribution

$$
P(E) d E=\frac{C}{E^{1+\mu}} d E
$$

with $E_{\min }=1 \leq E<\infty$; then $C=\mu$. Suppose that $N$ events occur within a given time interval. Let $E_{1} \geq E_{2} \geq \ldots \geq E_{n} \geq \ldots \geq E_{N}$ be the energies of the events listed in descending order. If $N$ is large, the power law distribution (1) is easily retrieved 
by the standard procedure of constructing a log-log plot of the number of events in a given energy interval. Suppose however that $N$ is small, let us say of the order of 10 or at most 100. In this case, the number of events will be much too small to permit the determination of $P(E)$ by standard techniques.

For illustrative purposes, consider the 100 largest events to be found in a random selection of $N=10^{5}$ samples from the power law distribution (1) with exponent $\mu=1$. We thus mimic the standard situation where only a fraction of the total population can be observed due to certain limitations of resolution. Our selection procedure has the same statistics as one in which we use $N=100, E_{\min }=1000$ and keep all 100 events generated. We plot $E_{n}$ as a function of $n$ (Fig. 1) for five realizations of the random process.

Figure 1 allows us to quantify our claim that the power law distribution can be extracted with reasonable precision from a few tens of the largest samples. A leastsquares fit to each of these five realizations in the interval $1 \leq n \leq 100$ shows that $E_{n}$ scales as $E_{n} \sim n^{-\gamma}$ with an exponent ranging from 0.85 to 1.22 . We can show that $\gamma=\frac{1}{\mu}$, which is easily obtained from the equation $N \int_{E_{n}}^{+\infty} P(E) d E \simeq n$; the latter constraint requires that on average there be $n$ values of $E$ larger than or equal to $E_{n}$ out of $N$ samples. Our estimate for $\mu$ thus ranges from 0.83 to 1.19 , which should be compared with the exact value $\mu=1$. If we increase the value of $N$, the precision of the determination of the exponent $\mu$ improves rapidly; if we increase $N$ to $N=10^{5}$, we get $\gamma=\mu=1.00 \pm 0.01$.

The rank-ordering technique is fundamentally different from attempts to improve the determination of the $b$-value from extreme-value statistics. This latter technique involves a discarding of a significant part of the data, and has been shown to be unreliable [Knopoff and Kagan, 1977]. The rank-ordering technique makes full use of all of the data available; we analyze it by studying the largest events.

The rank-order distribution is the same as the usual cumulative distribution but with an interchange of axes. However the statistical analyses of the two seemingly equivalent versions are significantly different: the statistics of the rank-ordering procedure focuses on the values of the energies or moments of the samples; the statistics of the cumulative distributions is concerned with uncertainties of the order numbers, which are integers. Rank-ordering statistics therefore provides a different perspective, with emphasis on the extreme tail of the distribution. We show that the rank-ordering 
statistics ensures that the fits are constrained by the largest events and not by the most numerous smaller ones.

\section{One power law: Analytical results}

Before proceeding with numerical tests of the rank-ordering method, we state the following results. The probability $F\left(E_{n}\right) d E_{n}$ that the energy for the nth order event be equal to $E_{n}$ within $d E_{n}$ is [Gumbel, 1960] :

$$
F\left(E_{n}\right) d E_{n}=\left(\begin{array}{c}
N \\
n
\end{array}\right)\left(1-\int_{E_{n}}^{+\infty} P(E) d E\right)^{N-n}\left(\begin{array}{c}
n \\
1
\end{array}\right)\left(\int_{E_{n}}^{+\infty} P(E) d E\right)^{n-1} P\left(E_{n}\right) d E_{n}
$$

for an arbitrary distribution $P(E)$. For the power law, $F\left(E_{n}\right)$ has a peak at $E_{n}^{\max }=$ $\left[\frac{(\mu N+1)}{\mu n+1}\right]^{1 / \mu}$, which recovers and makes precise the scaling law above. If $P(E)$ is given by the power law $(1), F\left(E_{n}\right)$ can be expanded around this maximum as

$$
F\left(E_{n}\right)=F\left(E_{n}^{\max }\right)-\frac{1}{2}\left|\frac{d^{2} F\left(E_{n}\right)}{d E_{n}^{2}}\right|_{E_{n}^{\max }}\left(E_{n}-E_{n}^{\max }\right)^{2}+\ldots,
$$

which allows us to get an estimate of the standard deviation of $E_{n}$ through the calculation of $\Delta E_{n} \equiv<\left(E_{n}-E_{n}^{\max }\right)^{2}>^{\frac{1}{2}}$, where the brackets indicate that the statistical average is taken. We obtain

$$
\frac{\Delta E_{n}}{E_{n}^{\max }}=\left(\frac{1}{\mu(n \mu+1)}\right)^{\frac{1}{2}}
$$

We have used this expression to construct the two straight lines in Fig. 1 that represent one standard deviation from the best fit. We recover the usual general result, also observed in Fig. 1, that $\frac{\Delta E_{n}}{\left\langle E_{n}\right\rangle}$ decays as $\frac{1}{\sqrt{ } n}$ for $n \gg 1$.

A maximum likelihood estimate for $\mu$ can be derived directly $[$ Aki, 1965; Deemer and Votaw, 1955; Hill, 1975]. Consider a variable $x$ that has the normalized exponential distribution $P(x)=\mu e^{-\mu x}, 0 \leq x<\infty$. Suppose that we measure only values of $x$ larger than some $x_{0}$ and that the total number of trials $N$ is not known. Then the probability distribution, restricted to have values larger than $x_{0}$, is $P_{\mu}(x)=\mu e^{-\mu\left(x-x_{0}\right)}$. For a set of $n$ samples $\left(x_{1}, x_{2}, \ldots, x_{n}\right)$ each greater than $x_{0}$ and 
having average value $\bar{x}$, the probability for this set is simply $P_{\mu}^{n}(x)=P_{\mu}\left(x_{1}\right) \ldots P_{\mu}\left(x_{n}\right)$, which can be written as $P_{\mu}^{n}(x)=\mu^{n} e^{-\mu n\left(\bar{x}-x_{0}\right)}$. The maximum likelihood estimate of the most probable value $\mu^{*}$ of $\mu$, is given by $\left.\frac{d P_{\mu}^{n}}{d \mu}\right|_{\mu^{*}}=0$, and is $\mu^{*}=\frac{1}{\bar{x}-x_{0}}$. Using rank-ordering for ranks 1 to $n$ thus yields the estimate $\mu=\frac{1}{\frac{1}{n} \sum_{i=1}^{n} x_{i}-x_{n}}$, where $x_{n}$ is the $n$th largest value. Under the change of variable $E=e^{x}, E$ is distributed according to a power law (1) with exponent $\mu$ if $x$ is exponentially distributed. Given the first $n$ largest values $E_{1} \geq E_{2} \geq \ldots \geq E_{n}$, the maximum likelihood estimate for the exponent $\mu$ is simply the maximum likelihood estimate of $\mu$ of the exponential distribution, expressed in terms of the variable $E$, since, if the probability is maximum for the exponential case, it is also maximum for $\mu$ with this monotonic increasing change of variable. Thus the maximum likelihood estimate for $\mu$ is $[A k i, 1965$; Deemer and Votaw, 1955; Hill, 1975]

$$
\mu=\frac{1}{\frac{1}{n} \sum_{i=1}^{n} \log \frac{E_{i}}{E_{n}}} .
$$

The accuracy of this estimate is given by the standard deviation $\Delta \mu=\left(\frac{d^{2} \log P_{\mu}^{n}}{d \mu^{2}}\right)^{\frac{1}{2}}$ which yields $\frac{\Delta \mu}{\mu}=\frac{1}{n^{1 / 2}}$, showing that the accuracy of the determination of $\mu$ improves according to the usual law of large numbers.

We can derive the full distribution of $\mu$. Let $\mu_{0}$ be the true value of the exponent. In order to derive the distribution of $\mu$ from a finite data sample, we note that

$$
<\log E>\left.\right|_{E>E^{*}}=\int_{E^{*}}^{+\infty} \log E P(E) d E=\log E^{*}+\frac{1}{\mu_{0}} .
$$

The quantity $\left\langle x>\left.\right|_{E>E^{*}}\right.$ is the average of $x$ with respect to its distribution, for those samples with $E$ conditioned to values larger than $E^{*}$. With eq. (4) which can also be written

$$
<\frac{1}{\mu}>\left.\right|_{E>E_{n}}=<\log E>\left.\right|_{E>E_{n}}-\log E_{n},
$$

this yields

$$
<\frac{1}{\mu}>\left.\right|_{E>E_{n}}=\frac{1}{\mu_{0}} .
$$

We next note that

$$
<(\log E)^{2}>\left.\right|_{E>E^{*}}=\left(\log E^{*}\right)^{2}+\frac{2 \log E^{*}}{\mu_{0}}+\frac{2}{\mu_{0}^{2}},
$$


which yields

$$
\operatorname{Var}[\log E]=<(\log E)^{2}>\left.\right|_{E>E^{*}}-<\log E>\left.^{2}\right|_{E>E^{*}}=\frac{1}{\mu_{0}^{2}} .
$$

With eq. (4), we obtain

$$
\operatorname{Var}\left[\frac{1}{\mu}\right]=\operatorname{Var}\left[\frac{\sum_{i=1}^{n} \log E_{i}-n \log E_{n}}{n}\right]=\frac{1}{n \mu_{0}^{2}} .
$$

The central limit theorem states that the distribution of $\sum_{i=1}^{n} \log E_{i}$ will have an approximate normal distribution for sufficiently large $n$. Thus, for known $\mu_{0}, \frac{1}{\mu}$ has an approximately normal distribution of mean $\frac{1}{\mu_{0}}$ and variance $\frac{1}{n \mu_{0}^{2}}$ :

$$
P\left(\frac{1}{\mu}\right)=\left(\frac{n}{2 \pi}\right)^{1 / 2} \mu_{0} e^{-\left(\frac{1}{\mu}-\frac{1}{\mu_{0}}\right)^{2} n \mu_{0}^{2} / 2} .
$$

The distribution $P(\mu)$ for $\mu$ is simply deduced by using the formula $P(\mu)=$ $P\left(\frac{1}{\mu}\right) \frac{d(1 / \mu)}{d \mu}$, yielding

$$
P(\mu)=\left(\frac{n}{2 \pi}\right)^{1 / 2} \frac{\mu_{0}}{\mu^{2}} e^{-\frac{n\left(\mu-\mu_{0}\right)^{2}}{2 \mu^{2}}}
$$

We note that the distribution $P(\mu)$ is skewed due to the prefactor $\frac{\mu_{0}}{\mu^{2}}$ in front of the exponential term. We discuss its practical importance below.

It is useful to give the maximum likelihood estimator for $\mu$ for the case where the data values are restricted in a finite range $E_{s} \leq E \leq E_{L}$. The previous case corresponds to $E_{L} \rightarrow+\infty$ and $E_{s}=E_{n}$. In the present case, we consider the possibility that, in some applications, the estimates of the higher values of magnitude may be unreliable, for instance their recordings may saturate the seismograph, or these estimates may be biased by finite size effects as may occur in some numerical simulations. In these cases, expression (4) should not be used but rather a modified version which takes into account the fact that values larger than some $E_{L}$ are not taken into account. A procedure that could be used in future work would be to examine the dependence of $\mu$ as a function of $E_{L}$. We start as above with the expression of the probability distribution for the exponential variable $x$, restricted to have values larger than $x_{s}: P_{\mu}(x)=\mu e^{-\mu\left(x-x_{s}\right)}, x_{s} \leq x<\infty$. Using the identity $P(A /$ knowing $B)=\frac{P(A)}{P(B)}$, the distribution restricted to have values $x$ between $x_{s}$ and $x_{L}$ is then simply

$$
P_{\mu}(x)=\frac{\mu e^{-\mu\left(x-x_{s}\right)}}{1-e^{-\mu x_{L}}}
$$


$x_{1}, x_{2}, \ldots, x_{n}$ are all between $x_{s}$ and $x_{L}$ with probability

$$
P_{\mu}\left(x_{1}\right) \ldots P_{\mu}\left(x_{n}\right)=\frac{\mu^{n} e^{-n \mu\left(\bar{x}-x_{s}\right)}}{\left(1-e^{-\mu x_{L}}\right)^{n}} .
$$

The maximum likelihood value for $\mu$ then obeys

$$
\frac{1}{\mu}-\left(\bar{x}-x_{s}\right)-\frac{1}{n} \frac{x_{L} e^{-\mu x_{L}}}{1-e^{-\mu x_{L}}}=0,
$$

which gives the previous result for $x_{L} \rightarrow+\infty$. With the change of variable $E=e^{x}$, we get the maximum likelihood estimate for the exponent $\mu$ from the knowledge of all the data values between $E_{s}$ and $E_{L}$ :

$$
\frac{1}{\mu}-\frac{1}{n} \sum_{i=1}^{n} \log \frac{E_{i}}{E_{s}}-\frac{1}{n} \frac{E_{L}^{-\mu} \log E_{L}}{1-E_{L}^{-\mu}}=0,
$$

which recovers eq. (4) for $E_{L} \rightarrow+\infty$ Deemer and Votaw, 1955].

These results highlight the power of the rank-ordering technique and its generalization to extract the power law distribution from the information contained in the largest events, which characterize the large-event tail of the distribution. The largest events constrain the tail of the distribution drastically and thus permit a surprisingly good recovery of the exponent from a relatively small data set. It is clear that the statistics improves if the number of the very largest events we consider increases, as shown by (3) and Fig. 1.

\section{Two power laws: Synthetic tests}

In view of our application of rank-ordering methods to earthquake data, we present tests of the method for synthetic distributions constructed from two power laws, each valid over a different range of energy. Let $P(E)=\frac{C_{1}}{E^{1+\mu_{1}}}$ for $1 \leq E \leq E_{c}$ and

$P(E)=\frac{C_{2}}{E^{1+\mu_{2}}}$ for $E_{c} \leq E<\infty$. The parameters $C_{1}$ and $C_{2}$ are determined from the normalization of $P(E)$ and the condition of continuity at $E_{c}$, for given $\mu_{1}, \mu_{2}$ and the cross-over value $E_{c}$. For a given number of events $N$, there is an exact relationship between the cross-over value $E_{c}$ and the average number of events $n_{2}$ that sample the power law for the larger events with $E>E_{c}$ :

$$
E_{c}=\left(1+\frac{\mu_{1}}{\mu_{2}}\left(\frac{N}{n_{2}}-1\right)\right)^{\frac{1}{\mu_{1}}}
$$


If the number of great events $n_{2}$ is large, corresponding to a not-too-large $E_{c}$, then the two power laws can be reconstructed easily through the standard log-log plot of the number of events in a given energy interval. However this will not be the case in the seismological example below. Therefore we consider the more difficult case where the number of large events $n_{2}$ is small, let us say of the order of a few tens of events.

Figure 2 shows the rank-ordering for a single realization with $N=10^{5}, \mu_{1}=\frac{2}{3}$, $\mu_{2}=\frac{4}{3}$ and $n_{2}=50$, corresponding to $E_{c} \sim 10^{5}$. (These choices for the exponents $\mu_{1,2}$ were made in view of values suggested in Pacheco, et al. [1992].) The two branches are clearly identifiable. The accuracy with which the exponent for the lower energy branch $\mu_{1}$ can be determined is of course excellent since the statistics covers more than 3 decades. However, since only about 50 extreme events sample the power law branch for large energies, there are large fluctuations in this branch, which raises the question whether there is a possibility to extract the exponent of the second power law and the cross-over energy from an analysis of the first tens of events with the largest energies in the extreme tail of the distribution. To determine the statistical accuracy of the value of the exponent $\mu_{2}$ for the largest events, we have generated $10^{5}$ different realizations with the parameters used in Fig. 2 and listed above. For each realization we select the $p$ largest events with $p=25,50,75,100$ and 200. For each $p$, we use eq. (4) to get an estimate of $\mu_{2}$ with $n$ running from 5 to $p$ to establish the stability of the determination of $\mu_{2}$. We have also made a least-square fit of the data $E_{n}$ with a power law over the $p$ points. In practice for this limited range $25 \leq p \leq 200$, the two methods give results which are essentially indistinguishable. However, the maximum likelihood estimate of eq. (4) is more sensitive to deviations from a pure power law. Although $n_{2}$ was fixed at 50 in the realizations, we assume that this number is not known in the analysis; thus we analyze the data as though we had no a priori information on the value of the cross-over, since this would not be generally known in earthquake data. The histogram of the number of realizations that have a given value of $\mu_{2, e s t}$ is shown in Fig. 3. The distribution of $\mu_{2, e s t}$ is significantly skewed and peaks at a value below the true value of $4 / 3$. The maximum value of $\mu_{2, \text { est }}$ increases progressively toward $4 / 3$ as $p$ increases up to around $p=75$ where it is close to 1.2 and then decreases for larger values of $p$; for $p=200$, the maximum is around 1.1. The latter decrease is due to the fact that the small-energy branch 
begins to influence significantly the determination of the slope of the large-energy branch if too many samples with rank orders $n>n_{2}$ are included, as can be observed directly from Fig. 2. The observed skewness and shift of the maximum are predicted from eq. (5), since the maximum of $P(\mu)$ occurs at $\mu=\mu_{0} \frac{2}{1+(1+[8 / p])^{1 / 2}}$, a value that is always smaller than the true value $\mu_{0}$. For example, if $p=25$, then $\mu=0.93 \mu_{0}$ and thus $\mu=1.24$, for $\mu_{0}=4 / 3$. Although this remark makes the existence of skewness plausible, the actual skewness is larger than predicted by eq. (5), which we attribute to the fact that equation (5) does not allow for additional fluctuations and distortions of the statistics due to the presence of the two power law branches in the distribution.

The curves in Fig. 3 are reminiscent of log-normal or Weibull distributions, being characterized by a sharp decay on one side and a long tail on the other side of the peak. Consider the Weibull distribution,

$$
p(\mu)=C m \mu^{m-1} e^{-C \mu^{m}}
$$

We can easily derive the two parameters $C$ and $m$ from the a priori knowledge of $<\mu>=\frac{4}{3}$ and from the direct observation of the most probable value $\mu_{x}$ which is the value at the maximum of the histogram of Fig. 3. We note in particular that the ratio $\frac{\langle\mu\rangle}{\mu_{x}}$ is a function solely of $m$. Thus we have a simple and direct determination of $m$ for each case $n=25,50,75,100,200$, which then allows us to estimate the width of the distribution, defined by the square root of its variance $V$. For instance, for $n=50$, we measure $\mu_{2} \simeq 1.08$, leading to $\frac{\langle\mu\rangle}{\mu_{2}} \simeq 1.25$. This is exactly the value expected for $m=2$ for which we compute $V^{\frac{1}{2}}=0.31$, in good agreement with inspection of Fig. 3 . Thus for $n=50$, we have $\mu_{2, \text { est }}=1.1 \pm 0.3$, which should be compared with the exact value $\mu_{2}=1.33$. For $n=75$ and 100 , we get $\mu_{2, e s t}=1.15 \pm 0.25(m \simeq 2.4)$. Thus we have quantified the skewness of the distribution: for our choice of parameters, the most probable measured value of $\left\{\mu_{2, e s t}\right\}_{x}$ is about 0.2 below the true value 1.33 , with a root-mean-square error of about 0.3 .

These results complement the simple maximum likelihood estimate derived above which states that the determination of $\mu_{2}$ has a relative error that is given by $\frac{\Delta \mu}{\mu}=$ $\frac{1}{n^{1 / 2}}$. For $n=50$ and $\mu_{2}=1.33$, this yields $\Delta \mu \simeq 0.19$, which is smaller than our numerical estimate of 0.3 . The synthetic tests have shown that there is a notable skewness of the same order of magnitude as the error. These results concerning the 
standard deviation and skewness are relevant to any interpretation of observations of earthquake data.

We also observe that, as $n$ becomes smaller, the probability that we measure a large value of $\mu_{2, \text { est }}$ becomes larger. For example, if $n=5$, the tail extends to $\mu_{2, \text { est }}=5$, and in about $5-10 \%$ of the realizations we find values of $\mu_{2, \text { est }}$ greater than 2. This reflects the large fluctuations in the energies for the lowest rank-order events which are those with the largest energies, as can be seen from (3). Using $\mu=4 / 3$, we find $\frac{\Delta E_{1}}{E_{1}^{x}} \simeq 0.66$ from (3), for the event with the largest energy.

We have also studied the properties of the rank-ordering technique for different values of $\frac{\mu_{2}}{\mu_{1}}$ and different $n_{2}$. We find that the larger the value of $n_{2}$, the easier and more precise the determination of $\mu_{2}$. However, for very small $n_{2} \sim 10$, there are some practical limitations. For instance, for $\mu_{1}=\frac{2}{3}$ and $\mu_{2}=1$, one can identify the presence of a cross-over, but the exponent $\mu_{2}$ that we derive is hugely overestimated by as much as a factor of 2 . On the other hand, if $\frac{\mu_{2}}{\mu_{1}}$ is of the order of or larger than 2 , the bias in the determination of $\mu_{2}$ approaches that of the determination of the exponent for a distribution without two branches, i.e. as though the distribution was that of a single power law with the exponent $\mu_{2}$, as measured from the few tens of events with the largest energies, as discussed above.

\section{Cross-over energies}

We consider the question of the determination of the cross-over values $n_{2}$ and $E_{c}$. Since we would like to understand the physical process responsible for the cross-over from the measurement of the cross-over value, in principle we should use a statistical method of interpretation which is independent of any a priori assumptions. This is a difficult problem, since the functional form of the cross-over is not known in general. It is clear from Fig. 2, for example, that the cross-over is not sharp, simply because of the cumulative nature of the rank-ordering procedure.

We have attempted to estimate $n_{2}$ by three different techniques. First, it is natural to use eq. 4 and plot $\mu$ as a function of $n$. For small $n$, one observes large fluctuations as expected. But typically, as soon as $n$ is of the order of $10, \mu$ enters a regime where it fluctuates around a constant average value with rough relative amplitudes $\frac{1}{\sqrt{n}}$. We might hope to detect the cross-over $n_{2}$ as corresponding to the rank $n^{*}$ beyond which 
a significant deviation from this constancy is observed. In those cases in which we did detect a bend or deviation from a constant value, we found that the corresponding rank $n^{*}$ had little to do with the average expected value $n_{2}=50$. Indeed, we observed $n^{*}$ to fluctuate typically between 20 or even less and about 100 .

A second attempt to estimate $n_{2}$ was made using the least-square fit method. We plotted the cost function, i.e. the rms of the residuals in the least-squares fit to the first $n$ rank-ordered samples, normalized by the number of events $n$. Our hope was that, for increasing $n<n_{2}$, the cost function would show a tendency to decrease as the large-energy branch of the distribution with exponent $\mu_{2}$ is approximated better and better. However, for $n$ larger than the true cross-over value $n_{2}$, the cost function would start to increase, and as $n$ becomes significantly larger than $n_{2}$, the fit to the overwhelming number of events in the smaller-energy branch should yield a single power law that would be insufficient to account for the cross-over between the two power laws; hence the cost function should deteriorate significantly. Thus we expect $n_{2}$ to be the value that minimizes the chi-square estimate. Among 1000 realizations with the same generating parameters listed above, we did not find any consistent behavior that would enable us to determine the cross-over value with good accuracy. Indeed, members of one subset of these of realizations have only a weak and often broad minimum in the cost function at values that differ from realization to realization in the range $n=5$ to 50 ; most realizations do not display any minimum whatsoever below $n=100$. In all cases, the cost function is extremely variable from realization to realization. We are forced to conclude that it is not possible to get a good estimate of $n_{2}$ by this method either.

In our third attempt to estimate the crossover, we used a less sophisticated method, namely we took the best estimate of $n_{2}$ to be the rank value at which the large-energy branch of the log-log rank-ordering plot begins to bend over by inspection. Because of the cumulative nature of the rank-ordering procedure, this definition is relatively precise; what is required is to identify the bendover. Other estimates of the cross-over, such as the intersection between the extrapolations of the two linear parts of the distribution are clearly biased. We have found that the estimate from the onset of bendover yields values which fluctuate between $n=10$ to 100 or more, while the value from which the data were synthesized is $n_{2}=50$. This wide range of estimates arises despite the existence of an apparent well-defined bend in the plots. 
Thus the appearance of a bend or kink on rank-order or cumulative plots should be interpreted with caution. We are led to suggest a warning: a given realization may exhibit an apparent cross-over at a rank-order value which has a large probability of being far from the true value by as much as a factor of two! Quantitatively, only about half the realizations have their apparent cross-over between 30 and 100 distributed around the true value of 50. Below, we comment on the relevance of these results to the earthquake problem.

\section{Application to earthquake catalogs}

We apply the rank-ordering technique to large earthquake catalogs. We address the problem of the determination of the Gutenberg-Richter magnitude-frequency law, which is often written as the cumulative distribution

$$
\log _{10} N_{>}=a-b M_{W}
$$

$N_{>}$is the number of earthquakes whose magnitudes are equal or greater than $M_{W}$, where $M_{W}$ is the magnitude, defined by

$$
M_{W}=\frac{1}{\beta}\left[\log _{10}\left(m_{0}\right)-9\right]
$$

where $m_{0}$ is the seismic moment in N.m; $\beta$ is generally taken equal to 1.5 . If we combine these two expressions, we get a power law distribution for the number of earthquakes having a given seismic moment that is identical to equation (1), charac-

terized by the exponent $\mu=\frac{b}{\beta}$. For small and intermediate magnitude earthquakes, $b \approx 1.0 ;$ thus $\frac{b}{\beta} \approx 2 / 3$.

We revisit the Harvard and the Southern California catalogs. The Harvard catalog we use [Dziewonski et al., 1993] spans the time interval from 1977 to 1992 for earthquakes worldwide, and the Southern California catalog, 1932-1991 [Hutton and Jones, 1993] spans almost 60 years of local seismicity. Both catalogs, as well as all other catalogs that span a broad range of magnitudes reliably, are dominated by the large numbers of small earthquakes; the time span of these catalogs is significantly 
shorter than the time interval between the strongest earthquakes, and as a consequence the data for the strongest earthquakes is sketchy at best. A search for a kink in the magnitude-frequency distribution has several hazards, the most obvious of which is the saturation in the magnitude scale, which taken literally, automatically generates a bend, if not a kink. Kanamori [1977] has used the seismic moment to derive a magnitude $M_{W}$ which is not susceptible to these saturation effects. For the Harvard catalog, we study the seismic moment distribution exclusively, and thereby also avoid errors due to the discretization of the magnitude scale; our accuracy is one order of magnitude greater than if we had used decimal magnitudes [Ekstrom and Dziewonski, 1988]. Hutton and Jones [1993] have recently re-evaluated many of the events in the Southern California catalog and have given values of the moment magnitude $M_{W}$.

In this section, we perform a statistical analysis of the scalar seismic moments $m_{0}$ in the Harvard catalog [Dziewonski et al., 1993], including moment entries that have become available since the publication of Pacheco et al. [1992]. In contrast with our numerical tests above, we no longer have the luxury of dealing with multiple realizations of the process: we can only treat real catalogs as the result of a single realization. Fig. 4 presents the logarithm of the nth-ordered seismic moment as a function of the logarithm of its rank, for the largest shallow earthquakes (defined as those whose hypocentral depths are less than $70 \mathrm{~km}$ ), in the Harvard catalog. Two regimes can be clearly distinguished: for the first 50 largest earthquakes, we get $\mu_{2}=1.3 \pm 0.1$, yielding $b_{2}=2.0 \pm 0.2$ assuming $\beta=1.5$, for the large-energy branch. Here, the error bars are estimated from the least-squares fit procedure and do not reflect the statistical error inherent in a single realization. From the results obtained in section 3, in particular taking into account the existence of a skewness and intrinsic statistical error, we correct this raw result and write instead $\mu_{2}^{\text {corrected }}=$ $1.5 \pm 0.2$ leading to $b_{2}=2.3 \pm 0.3$, a value to be compared with the earlier estimate of $b_{2}=1.6 \pm 0.1$ [Pacheco et al., 1992]. A value of $b_{2}$ near 2.3 is wholly consistent with the argument that the earthquake energy flux is finite [Knopoff and Kagan, 1977; Kagan and Knopoff, 1984; Kagan, 1994]; however, it is quite inconsistent with the values suggested for either a model wherein the moment of large ruptures is controlled by the width of the rupture (W-model) or by its length (L-model) derived from simple scaling arguments Romanowicz and Rundle, 1993]. A value of $b_{2} \simeq 2.3$ appears to be 
more compatible with a $\mathrm{W}$-model on the basis of a recent calculation of the exponent by means of a nonlinear diffusion equation [Sornette and Sornette, 1995] which yields $b_{2} \simeq 3$.

For much larger ranks that correspond to the smaller earthquakes in the catalog, we obtain $b_{1}=1.00 \pm 0.02(\mu=0.67 \pm 0.01)$ in rough agreement with the value given by Pacheco, et al. [1992] $b_{1}=1.07 \pm 0.01$. Note that this power law for small events has a stable exponent only if we use very large ranks, from around 200 and above; we must avoid using data near the apparent cross-over; the instability in the exponent always appears if we have two regimes for the distribution (see Fig. 2). It would thus be erroneous to attempt to extract a second exponent for ranks between say 50 and 200. Of course, the same conclusion is valid for the cumulative distribution. Using the results of Deemer and Votaw [1955], Kagan [1994] evaluated the possibility that a distribution with two power-law exponents could approximate the Harvard data in the magnitude interval $5.8 \geq M_{W} \geq 7.8$, and found that there is no statistically significant crossover at this magnitude range.

From our tests on synthetic data sets, we have shown that the determination of the cross-over value has a poor accuracy. It should be stressed that this is true even when the particular realization under study exhibits an apparent well-defined crossover value, as is the case for the Harvard earthquake catalog. Following Pacheco et al. [1992], one could read on the plot the value $n_{2} \simeq 50$ and conclude therefore that there is a well-defined cross-over magnitude at $M_{W} \simeq 7.5$, in agreement with the Pacheco et al. result of $M_{W} \simeq 7.4$. We have noted the danger in this identification when tested on synthetic data sets: the existence of intrinsic statistical fluctuations makes the apparent cross-over fluctuate within a factor two of the true value from realization to realization. Thus our statistical tests have shown that any claim of a good determination of a cross-over must be tempered and that the true cross-over has a probability $1 / 2$ to be between rank 30 and rank 100. Translated into magnitudes, there is a probability of $1 / 2$ that the cross-over lies in the interval $7.1<M_{W}<7.6$, an interval that corresponds to a down-dip thickness $W$ of about 36 to $62 \mathrm{~km}$, values derived from the formula $W \sim\left(m_{0} / \Delta \sigma\right)^{1 / 3}$ with a coefficient given by [Pacheco et al., 1992]; $\Delta \sigma$ is the stress drop.

We point out that the data available to date are not sufficient in numbers to certify the existence of a power law distribution for large earthquakes. We have found 
that a gamma distribution [Kagan, 1994] also represents the data well and accurately. The gamma distribution has only two adjustable parameters; a distribution with two power-law branches has three adjustable parameters, and on grounds of statistical parsimony, a data fitting scheme with a smaller number of parameters is to be preferred, unless there is some compelling reason to use a more complex fitting scheme. There is no doubt that there is a rollover or cross-over to a distribution that falls off at a faster rate than for the smaller earthquakes. We have shown here that if we assume it to be a cross-over to another power law, then we can estimate the value of its exponent reasonably well in the case of the Harvard catalog; we cannot estimate the cross-over moment or magnitude with any reasonable certainty.

Fig. 5 shows the nth-ordered magnitude $M_{W}$ as a function of the logarithm of its rank for the Southern California catalog [Hutton and Jones, 1993]. A single power law fits the whole range. Since the magnitude (and not the logarithm of the moment) is plotted, the slope of the straight line gives $\frac{1}{b}$ directly, yielding $b=1.00 \pm 0.02$. We thus conclude that, in contrast with the results for the world-wide catalog and previous claims [Pacheco et al., 1992], the statistics for the Southern California catalog is fully compatible with a single power law distribution. We see no evidence for a bend or kink in the curve at $M=5.9$ to 6.0. This result causes us to question the validity of the proposal [Pacheco et al., 1992] that the thickness of the seismogenic zone determines the cross-over magnitude for Southern California, and thus we may expect that there is no observable influence of source dimension on the frequency distribution of earthquakes in other localities as well.

\section{The next big earthquake}

We comment that the rank-ordering technique may be used to infer the size of the next forthcoming biggest event. If the forthcoming large event is assumed to be imminent, its occurrence corresponds to a shift of the largest event recorded to date to rank 2, the second largest event to date to rank 3, and so on. A fit of the rankordering plot extrapolated to $n=1$ yields the seismic moment of the next very largest earthquake. Performing this analysis on the Southern California catalog, we obtain that the next largest event should be of magnitude $M_{W}$ around $7.9 \pm 0.2$. The same analysis applied to the Harvard catalog suggests that the next greatest 
earthquake immediately larger than any in the catalog should have a seismic moment $m_{0} \simeq 6 \cdot 10^{21} \mathrm{Nm}$, i.e. $M_{W} \simeq 8.5 \pm 0.2$. The uncertainties have been estimated using eq. (3).

We can be more precise and use the maximum likelihood formalism described above to infer the most probable value $E^{\text {next }}$ of the energy of the next earthquake, restricted to be larger than some threshold $E^{*}$ corresponding the present rank $n$ (i.e. such that $E^{*}=E_{n}$ ). In other words, we are discussing here restricted most probable values. To do this calculation, we make use of eq. (4) for the $n$th and $(n+1)$ st events having energies larger than $E^{*}=E_{n}$, assuming that $n$ is sufficiently large, so that $1 / \mu$ is close to its true value. In this case

$$
1 / \mu=\frac{1}{n} \sum_{i=1}^{n} \log \frac{E_{i}}{E_{n}}=\frac{1}{n} \sum_{i=1}^{n+1} \log \frac{E_{i}^{\prime}}{E_{n}},
$$

where the $E_{i}^{\prime}=E_{i}$ for $E_{i}>E^{\text {next }}$ and $E_{i+1}^{\prime}=E_{i}$ for $E_{i}<E^{\text {next }}$. Thus the most probable value of the next earthquake with $E$ larger than $E^{*}$ is $E^{n e x t}=E^{*} e^{\frac{1}{\mu}}$. This means roughly that the next event will have the $\left(\frac{n}{e}\right)$ th rank. In other words, the next event with $E$ larger than some threshold $E^{*}=E_{n}$ will not be, in general, the largest of all, but only larger than the threshold by a finite factor. If we stretch this argument to the unreal limit $n=1$ in the above formula, we can determine the most probable value of the next earthquake, with $E$ larger than the largest observed to date, $E_{1}$. We get $E^{\text {next }}=\frac{E_{1}}{E_{2}} E_{1}$ which is equal on average to $E^{\text {next }}=\left(\frac{2 \mu+1}{\mu+1}\right)^{\frac{1}{\mu}} E_{1}=2.3 E_{1}$ for $\mu=2 / 3$ corresponding to a magnitude gap of $0.6 \pm 0.3$; the uncertainty is estimated from eq. (3). We can ask a similar question about the energy of the next earthquake such that its energy will be larger than the second largest earthquake observed to date. The result is $E^{n e x t}=\left(\frac{E_{1}}{E_{2}}\right)^{1 / 2} E_{2}$. And so on.

\section{Summary}

The well-known rank-ordering statistical technique is useful for extracting the tail of the distribution for a sparse data set. The combination of analytical results and numerical tests has allowed us to quantify the accuracy that can be obtained for the determination of the exponents of power law distributions. We have confirmed that two power law branches to the distribution might account for the moments in the Harvard seismic moment catalog and we have corrected our estimate of the two 
corresponding $b$-values for skewness and intrinsic statistical errors. However, the cross-over value between the two power laws has been shown to be ill-defined. In the case of the Southern California catalog, we have shown that a single power law distribution, without any large-energy branch satisfies the observations; we find no evidence for a bend or kink in the distribution, nor can we make any inference about the thickness of the seismogenic zone from the earthquake size distribution.

We believe that this technique will be useful to analyze other geological data sets, often characterized by undersampled fat tails that correspond to rare extreme events.

\section{Acknowledgments}

D.S. acknowledges stimulating discussions with J.-P. Bouchaud. This work has been partially supported by the CNRS-NSF International Cooperation program, and by the Southern California Earthquake Center. Publication No. XXXX, Institute of Geophysics and Planetary Physics, University of California, Los Angeles. Publication No. YYYY, Southern California Earthquake Center. 


\section{References}

- Aharony A. and J. Feder eds., Fractals in Physics, North Holland, Amsterdam, Physica D, 38, nos. 1-3, 1989.

- Aki K., Maximum likelihood estimate of $b$ in the formula $\log N=a-b m$ and its confidence limits, Bull. Earthquake Res. Inst. Tokyo Univ., 43, 237-239 (1965).

- Deemer W.L. and D.F. Votaw, Estimation of parameters of truncated or censored exponential distributions, Ann. Math. Stat., 26, 498-504, 1955.

- Dziewonski A.M., G. Ekstrom, and M. P. Salganik, Centroid-moment tensor solutions for January-March, 1992, Phys. Earth Planet. Inter., 77, 143-150, 1993, and references therein.

- Ekstrom G. and A. M. Dziewonski, Evidence of bias in estimation of earthquake size, Nature, 332, 319-323, 1988.

- Epstein B. and M. Sobel, Life testing, J. Amer. Statist. Assoc., 48, 486-502, 1953.

- Geller R.J., Scaling relations for earthquake source parameters and magnitudes. Bull. Seismol. Soc. Amer., 66, 1501-1523, 1976.

- Gumbel E.J., Statistics of Extremes, Columbia Univ. Press, New York, 1960.

- Hill, B.M. A simple general approach to inference about the tail of a distribution, Ann. Statistics, 3, 1163-1174, 1975.

- Hutton L.K. and L.M. Jones, Local magnitudes and apparent variations in seismicity rates in Southern California, Bull. Seismol. Soc. Amer., 83, 313329, 1993, and references therein.

- Kagan Y.Y., Observational evidence for earthquakes as a nonlinear dynamic process, Physica D, 77, 160-192, 1994. Also appeared in Modeling the Forces of Nature, eds. R. Camassa, J. M. Hyman, and W. I. Newman, North-Holland, Amsterdam, 160-192, 1994. 
- Kagan Y.Y. and L. Knopoff, A stochastic model of earthquake occurrence, Proc. 8th Int. Conf. Earthq. Eng., 1, 295-302, 1984.

- Kanamori H., The energy release in great earthquakes, J. Geophys. Res., 82, 2981-2987, 1977.

- Kanamori H. and D.L. Anderson, Theoretical basis of some empirical relations in seismology, Bull. Seism. Soc. Am., 65, 1073-1095, 1975.

- Knopoff L. and Y. Kagan, Analysis of the theory of extremes as applied to earthquake problems, J. Geophys. Res. 82, 5647-5657, 1977.

- Mandelbrot B.B., The Fractal Geometry of Nature, Freeman, New York, 1983.

- Okal E.A. and B.A. Romanowicz, On the variation of $b$-values with earthquake size. Phys. Earth Planet. Inter., 87, 55-76, 1994.

- Pacheco J.F., C.H. Scholz and L.R. Sykes, Changes in frequency-size relationship from small to large earthquakes, Nature, 355, 71-73, 1992.

- Riste T. and D. Sherrington eds., Spontaneous formation of space-time structures and criticality, Proc. NATO ASI, (Geilo, Norway), Kluwer, Dordrecht, 1991.

- Romanowicz B., Strike-slip earthquakes on quasi-vertical transcurrent faults: inferences for general scaling behavior, Geophys. Res. Lett., 19, 481-484, 1992.

- Romanowicz B. and J.B. Rundle, On scaling relations for large earthquakes, Bull. Seism. Soc. Am., 83, 1294-1297, 1993.

- Rundle J.B., Derivation of the complete Gutenberg-Richter magnitudefrequency relation using the principle of scale invariance, J. Geophys. Res., 94, 12,337-12,342, 1989.

- Scholz C., The mechanics of Earthquakes and Faulting, Cambridge University Press, Cambridge, 1990.

- Shimazaki K., Small and large earthquakes: The effects of the thickness of seismogenic layer and the free surface. In Earthquake Source Mechanics, S. Das, 
J. Boatwright and C. Scholz, eds., Geophys. Monograph 37, Amer. Geophys. Un.,Washington, 1986, pp 209-216.

- Sornette D. and A. Sornette, Theoretical implications of a recent non-linear diffusion equation linking short-time deformation to long-time tectonics, Bull. Seism. Soc. Am., in press, 1995.

- Zipf G.K., Human Behavior and the Principle of Least-effort, Addison-Wesley, Cambridge, 1949. 


\section{FIGURE CAPTIONS:}

Fig. 1. Rank-order distribution for five different realizations of a random process whose values are distributed according to the power law (1) with $\mu=1 . N=10^{5}$ events were generated numerically for each realization and the 100 largest events were retained to construct the distributions. The two straight lines represent the expected standard deviation interval obtained from (3).

Fig. 2 Rank-ordering of a single simulation run with parameters $N=10^{5}, \mu_{1}=\frac{2}{3}$,

$\mu_{2}=\frac{4}{3}$ and $n_{2}=50$, corresponding to $E_{c} \sim 10^{5}$. The two power law branches of the distribution are clearly identifiable.

Fig. 3 Histogram of the number of realizations with the same $\mu_{2, \text { est }}$ out of $10^{5}$ realizations. Each realization was generated to have $N=10^{5}$ events, $\mu_{1}=\frac{2}{3}, \mu_{2}=\frac{4}{3}$, and cross-over value $n_{2}=50$ (i.e. $E_{c} \sim 10^{5}$ ). The five curves correspond to different values of $n(n=25,50,75,100$ and 200) which is the interval over which the estimate of the exponent $\mu_{2, e s t}$ is extracted.

Fig. 4 Log-log plot of the rank-ordered seismic moment (in units of $10^{19} \mathrm{Nm}$ ) of the largest shallow earthquakes $(h<70 \mathrm{~km})$ in the Harvard catalog versus its rank.

Fig. 5 The rank-ordered magnitude $M_{W}$ of the strongest earthquakes in the Southern California catalog vs. the logarithm of its rank. 